\title{
3D Matrix Ring with a “Common” Multiplication
}

\author{
Orgest Zaka \\ Department of Mathematics, Faculty of Technical Science, University of Vlora "Ismail QEMALI", Vlora, Albania \\ Email: gertizaka@yahoo.com
}

How to cite this paper: Zaka, O. (2017) 3D Matrix Ring with a "Common" Multiplication. Open Access Library Journal, 4: e3593.

https://doi.org/10.4236/oalib.1103593

Received: April 11, 2017

Accepted: May 12, 2017

Published: May 15, 2017

Copyright (๑) 2017 by author and Open Access Library Inc.

This work is licensed under the Creative Commons Attribution International License (CC BY 4.0).

http://creativecommons.org/licenses/by/4.0/ (c) (i) Open Access

\begin{abstract}
In this article, starting from geometrical considerations, he was born with the idea of 3D matrices, which have developed in this article. A problem here was the definition of multiplication, which we have given in analogy with the usual $2 \mathrm{D}$ matrices. The goal here is $3 \mathrm{D}$ matrices to be a generalization of $2 \mathrm{D}$ matrices. Work initially we started with $3 \times 3 \times 3$ matrix, and then we extended to $m \times n \times p$ matrices. In this article, we give the meaning of $3 \mathrm{D}$ matrices. We also defined two actions in this set. As a result, in this article, we have reached to present 3-dimensional unitary ring matrices with elements from a field F.
\end{abstract}

\section{Subject Areas}

Algebra, Applied Statistical Mathematics, Geometry

\section{Keywords}

Linear Algebra, Matrices, Ring Theory

\section{Introduction}

Based on the meaning of the addition and the multiplication of $2 \mathrm{D}$ matrices [1]-[6], this article stretches this sense, the idea, the addition and the multiplication of 3D matrices. Starting from geometrical considerations, concretely taking into account the cube, he was born with the idea of 3D matrices, which have developed in this article. A problem here was the definition of multiplication, for which we have acted pages, analogously acted as the columns, which we have given in analogy with the usual 2D matrices [6] [9]. The goal here is $3 \mathrm{D}$ matrices to be a generalization of $2 \mathrm{D}$ matrices. We proved that this set of two actions together in forming the "unitary ring" [7] [8] [10] [11]. In literature and in various mathematical forums, we noticed an interest in the $3 \mathrm{D}$ matrices, but on the other hand are missing results associated with them; this was a sufficient reason to explore. We introduced the meaning of the scalar multiplication, and finally 
we have shown that we have an $F$-module connected to this ring 3D matrices or vector spaces [8] [10]. As indications for this paper were simply geometric imaginations. Everything presented in this article are my results.

\section{Addition of $3 \times 3 \times 3$, 3-D Matrices over Field $F$, and the Addition Abelian Group of Their 3-D Matrices}

Imagining a parallelepiped, with born idea of 3D matrices, which are define as follows

Definition 2.1 3-dimensional $3 \times 3 \times 3$ matrice will call, a matrix which has: three horizontal layers (analogous to three rows), three vertical page (analogue with three columns in the usual matrices) and three vertical layers two of which are hidden.

The set of these matrices the write how:

$$
\mathcal{M}_{3 \times 3 \times 3}(F)=\left\{\left(a_{i j k}\right) \mid a_{i j k} \in F \text { and } i=1,2,3 ; j=1,2,3 ; k=1,2,3\right\}
$$

The appearance of these matrices will be as in Figure 1.

Definition 2.2 The addition of two matrices $A_{3 \times 3 \times 3}, B_{3 \times 3 \times 3} \in \mathcal{M}_{3 \times 3 \times 3}(F)$ we will call the matrix:

$$
C_{3 \times 3 \times 3}=\left\{\left(c_{i j k}\right) \mid c_{i j k}=a_{i j k}+b_{i j k}, \forall i, j, k \in\{1,2,3\}\right\}
$$

The appearance of the addition of $3 \times 3 \times 3$, 3D matrices, will be as in Figure 2, where matrices $\boldsymbol{A}$ and $\boldsymbol{B}$ have the following appearance,

$$
\begin{aligned}
& \boldsymbol{A}_{3 \times 3 \times 3}=\left\{\left(a_{i j k}\right) \mid a_{i j k} \in F \text { for } i=1,2,3 ; j=1,2,3 ; k=1,2,3\right\} \\
& \boldsymbol{B}_{3 \times 3 \times 3}=\left\{\left(b_{i j k}\right) \mid b_{i j k} \in F \text { for } i=1,2,3 ; j=1,2,3 ; k=1,2,3\right\}
\end{aligned}
$$

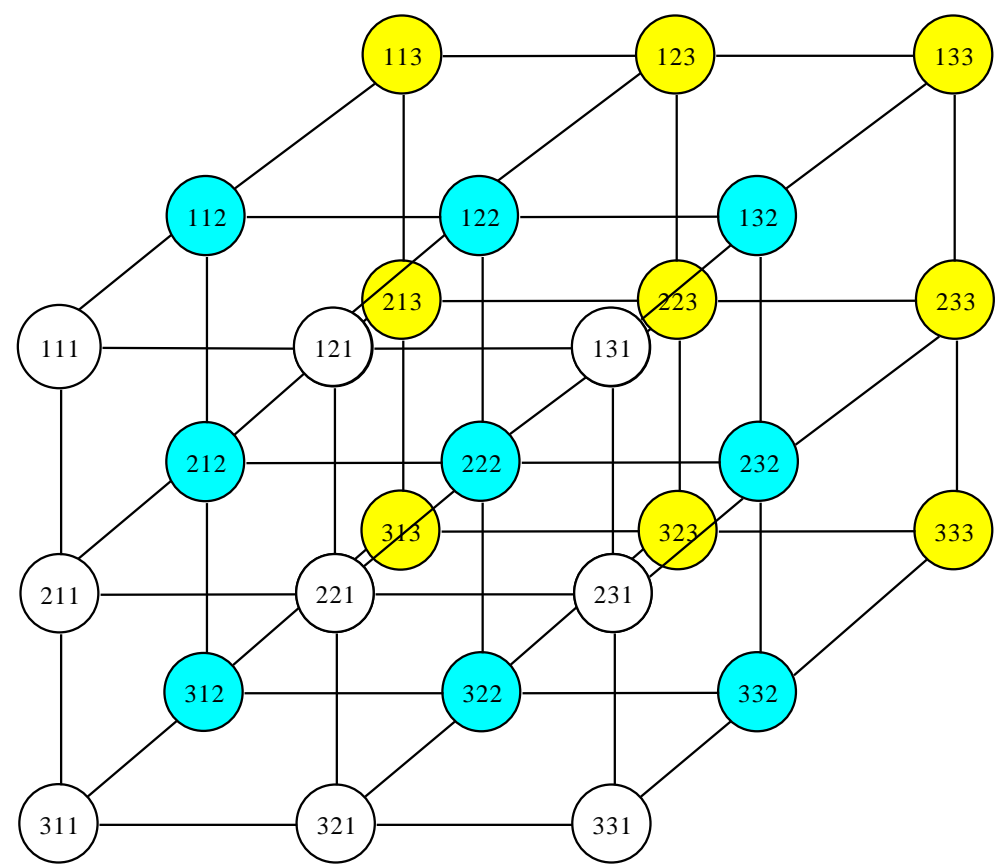

Figure 1. 3-D Matrice. 

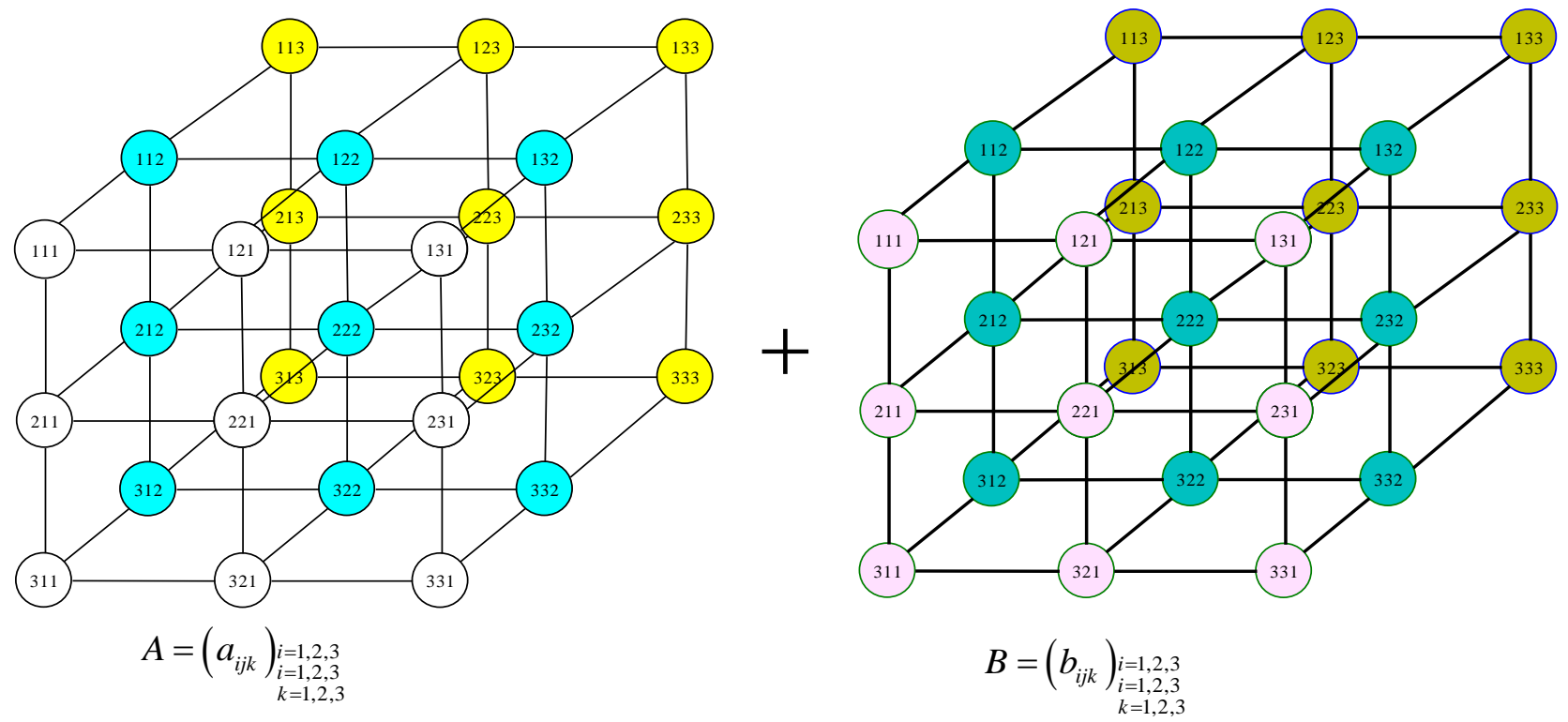

Figure 2 . The addition of $3 \times 3 \times 3,3 \mathrm{D}$ matrices.

Definition 2.3 Zero matrix $3 \times 3$, 3D we will called the matrix that has all its elements zero.

$$
\boldsymbol{O}_{3 \times 3 \times 3}=\left\{\left(0_{F}\right)_{i j k} \mid i=1,2,3 ; j=1,2,3 ; k=1,2,3\right\}
$$

Definition 2.4. The opposite matric of anmatrice

$$
A_{3 \times 3 \times 3}=\left\{\left(a_{i j k}\right) \mid i=1,2,3 ; j=1,2,3 ; k=1,2,3\right\}
$$

will, called matrix

$$
-\boldsymbol{A}_{3 \times 3 \times 3}=\left\{\left(-a_{i j k}\right) \mid i=1,2,3 ; j=1,2,3 ; k=1,2,3\right\}
$$

(where $-a_{i j k}$ is a opposite element of element $a_{i j k} \in F$, so $a_{i j k}+\left(-a_{i j k}\right)=0_{F}$ and $(F,+, \cdot)$ is field [8] [10] [11]), which satisfies the condition

$$
\begin{aligned}
\boldsymbol{A}_{3 \times 3 \times 3}+\left(-\boldsymbol{A}_{3 \times 3 \times 3}\right) & =\left\{\left(a_{i j k}+\left(-a_{i j k}\right)\right) \mid i=1,2,3 ; j=1,2,3 ; k=1,2,3\right\} \\
& =\left\{(0)_{i j k} \mid i=1,2,3 ; j=1,2,3 ; k=1,2,3\right\}=\boldsymbol{O}_{3 \times 3 \times 3}
\end{aligned}
$$

Theorem $2.1\left(\mathcal{M}_{3 \times 3 \times 3}(F),+\right)$ is a beliangrup.

Proof: Truly from the definition 2.2, of addition the 3-Dmatrices, we see that addition is the sustainable in $\mathcal{M}_{3 \times 3 \times 3}(F)$, because

$$
a_{i j k} \in F, b_{i j k} \in F \Rightarrow c_{i j k}=a_{i j k}+b_{i j k} \in F, \forall i, j, k \in\{1,2,3\}
$$

1) Associative property,

$$
\forall \boldsymbol{A}=\left(a_{i j k}\right), \boldsymbol{B}=\left(b_{i j k}\right), \boldsymbol{C}=\left(c_{i j k}\right) \in \mathcal{M}_{3 \times 3 \times 3}(F) \Rightarrow(\boldsymbol{A}+\boldsymbol{B})+\boldsymbol{C}=\boldsymbol{A}+(\boldsymbol{B}+\boldsymbol{C})
$$

truly

$$
\begin{aligned}
(\boldsymbol{A}+\boldsymbol{B})+\boldsymbol{C} & =\left[\left(a_{i j k}\right)+\left(b_{i j k}\right)\right]+\left(c_{i j k}\right)=\left(a_{i j k}+b_{i j k}\right)+\left(c_{i j k}\right)=\left(\left(a_{i j k}+b_{i j k}\right)+c_{i j k}\right) \\
& =\left(a_{i j k}+b_{i j k}+c_{i j k}\right)=\left(a_{i j k}+\left(b_{i j k}+c_{i j k}\right)\right)=\left(a_{i j k}\right)+\left(b_{i j k}+c_{i j k}\right)=\boldsymbol{A}+(\boldsymbol{B}+\boldsymbol{C})
\end{aligned}
$$


2) $\forall \boldsymbol{A}=\left(a_{i j k}\right) \in \mathcal{M}_{3 \times 3 \times 3}(F), \exists \boldsymbol{O}=\left(0_{i j k}\right) / \boldsymbol{A}+\boldsymbol{O}=\boldsymbol{O}+\boldsymbol{A}=\boldsymbol{A}$.

truly, $\forall \boldsymbol{A}=\left(a_{i j k}\right) \in \mathcal{M}_{3 \times 3 \times 3}(F), \exists \boldsymbol{O}=\left(0_{i j k}\right) / \boldsymbol{A}+\boldsymbol{O}=\boldsymbol{O}+\boldsymbol{A}=\boldsymbol{A}$.

$$
\begin{aligned}
\boldsymbol{A}+\boldsymbol{O} & =\left\{\left(a_{i j k}\right)+(0)_{i j k} \mid i=1,2,3 ; j=1,2,3 ; k=1,2,3\right\} \\
& =\left\{\left(a_{i j k}+0\right)_{i j k} \mid i=1,2,3 ; j=1,2,3 ; k=1,2,3\right\} \\
& =\left\{\left(a_{i j k}\right) \mid i=1,2,3 ; j=1,2,3 ; k=1,2,3\right\}=\boldsymbol{A}
\end{aligned}
$$

3) $\forall \boldsymbol{A}=\left(a_{i j k}\right) \in \mathcal{M}_{3 \times 3 \times 3}(F), \exists-\boldsymbol{A}=\left(-a_{i j k}\right) \in \mathcal{M}_{3 \times 3 \times 3}(F) / \boldsymbol{A}+(-\boldsymbol{A})=\boldsymbol{O}$. truly, from Definition 2.4, we have

$$
\begin{aligned}
\boldsymbol{A}+(-\boldsymbol{A}) & =\left\{\left(a_{i j k}+\left(-a_{i j k}\right)\right) \mid i=1,2,3 ; j=1,2,3 ; k=1,2,3\right\} \\
& =\left\{(0)_{i j k} \mid i=1,2,3 ; j=1,2,3 ; k=1,2,3\right\}=\boldsymbol{O}
\end{aligned}
$$

4) Addition is commutative.

$$
\forall \boldsymbol{A}=\left(a_{i j k}\right), \boldsymbol{B}=\left(b_{i j k}\right) \in \mathcal{M}_{3 \times 3 \times 3}(F), \boldsymbol{A}+\boldsymbol{B}=\boldsymbol{B}+\boldsymbol{A} .
$$

truly

$$
\boldsymbol{A}+\boldsymbol{B}=\left(a_{i j k}\right)+\left(b_{i j k}\right)=\left(a_{i j k}+b_{i j k}\right) \stackrel{(\mathbb{R},+) \text { is abelian }}{=}\left(b_{i j k}+a_{i j k}\right)=\left(b_{i j k}\right)+\left(a_{i j k}\right)=\boldsymbol{B}+\boldsymbol{A}
$$

\section{Addition of $m \times n \times p, 3$-D Matrices over Any Field $F$ and the Addition Abelian Group of Their 3-D Matrices}

Definition 3.1 3-dimensional $m \times n x p$ matrix will call, a matrix which has: $m$ horizontal layers (analogous to m-rows), n-vertical page (analogue with $\mathrm{n}$-columns in the usualmatrices) and $p$-vertical layers ( $p-1$ of which are hidden).

The set of these matrixes the write how:

$$
\mathcal{M}_{m \times n \times p}(F)=\left\{\left(a_{i j k}\right) \mid a_{i j k} \in F \text {-field and } i=\overline{1, m} ; j=\overline{1, n} ; k=\overline{1, p}\right\}
$$

Definition 3.2 The addition of two matrices $A, B \in \mathcal{M}_{m \times n \times p}(F)$ we will call the matrix:

$$
C_{m \times n \times p}=\left\{\left(c_{i j k}\right) \mid c_{i j k}=a_{i j k}+b_{i j k}, \forall i=\overline{1, m} ; j=\overline{1, n} ; k=\overline{1, p}\right\}
$$

The appearance of the addition of mxnxp, 3D matrices will be as in Figure 3, where matrices $\boldsymbol{A}$ and $\boldsymbol{B}$ have the following appearance,

$$
\begin{aligned}
& \boldsymbol{A}_{m \times n \times p}=\left\{\left(a_{i j k}\right) \mid i=\overline{1, m} ; j=\overline{1, n} ; k=\overline{1, p}\right\} \\
& \boldsymbol{B}_{m \times n \times p}=\left\{\left(b_{i j k}\right) \mid i=\overline{1, m} ; j=\overline{1, n} ; k=\overline{1, p}\right\}
\end{aligned}
$$

Definition 3.3 3-D, Zero matrix $m \times n \times p$, we will called the matrix that has all its elements zero.

$$
\boldsymbol{O}=\boldsymbol{O}_{m \times n \times p}=\left\{(0)_{i j k} \mid i=\overline{1, m} ; j=\overline{1, n} ; k=\overline{1, p}\right\}
$$

Definition 3.4 The opposite matric of anmatrice

$$
A_{m \times n \times p}=\left\{\left(a_{i j k}\right) \mid i=\overline{1, m} ; j=\overline{1, n} ; k=\overline{1, p}\right\} \in \mathcal{M}_{m \times n \times p}(F)
$$

will, called matrix 

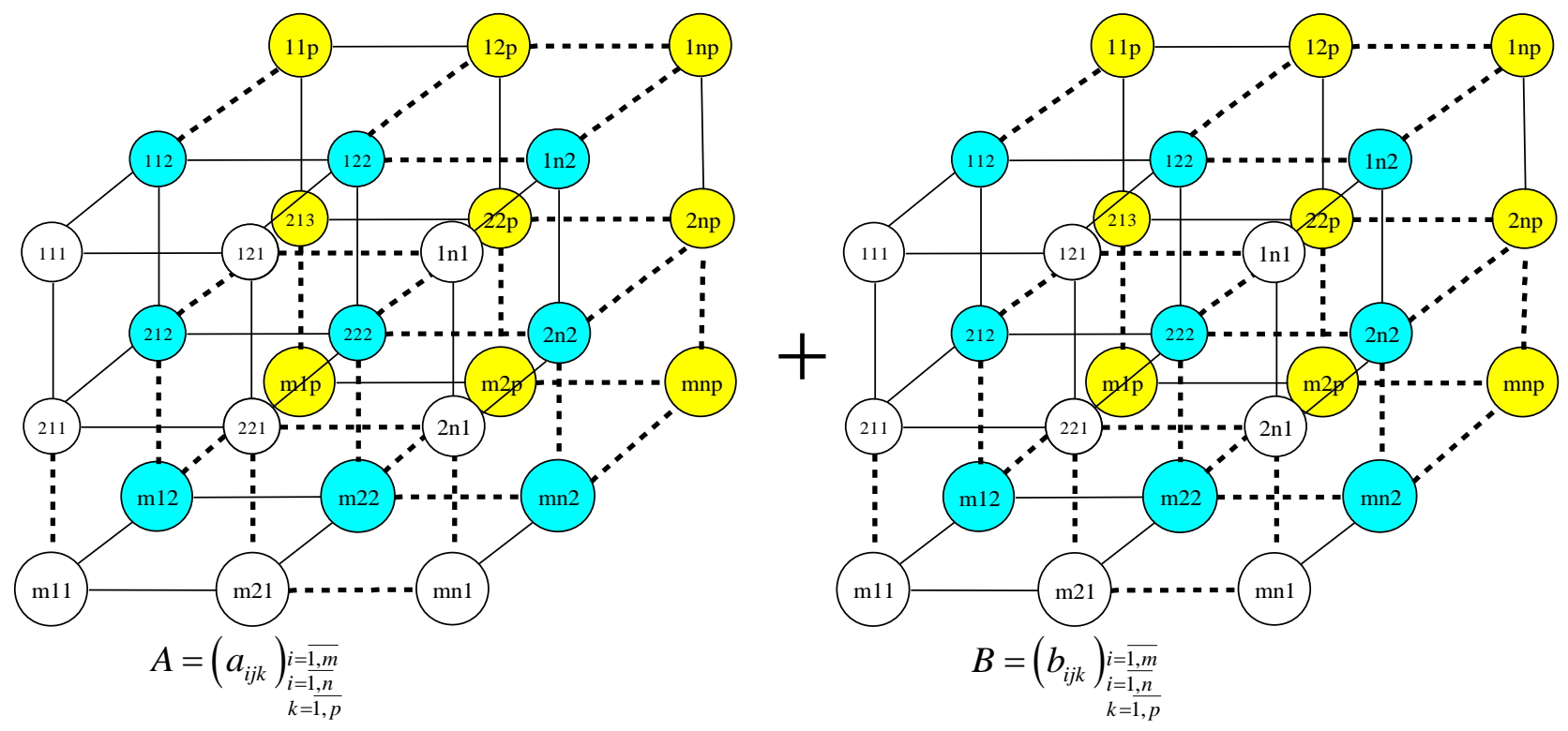

Figure 3. The addition of mxnxp, 3D matrices.

$$
-\boldsymbol{A}_{m \times n \times p}=\left\{\left(-a_{i j k}\right) \mid i=\overline{1, m} ; j=\overline{1, n} ; k=\overline{1, p}\right\} \in \mathcal{M}_{m \times n \times p}(F)
$$

(where $-a_{i j k}$ is a opposite element of element $a_{i j k} \in F$, so $a_{i j k}+\left(-a_{i j k}\right)=0_{F}$ and $(F,+, \cdot)$ is field), which satisfies the condition

$$
\begin{aligned}
\boldsymbol{A}_{m \times n \times p}+\left(-\boldsymbol{A}_{m \times n \times p}\right) & =\left\{\left(a_{i j k}+\left(-a_{i j k}\right)\right) \mid i=\overline{1, m} ; j=\overline{1, n} ; k=\overline{1, p}\right\} \\
& =\left\{(0)_{i j k} \mid i=\overline{1, m} ; j=\overline{1, n} ; k=\overline{1, p}\right\}=\boldsymbol{O}
\end{aligned}
$$

Theorem $3.1\left(\mathcal{M}_{m \times n \times p}(F),+\right)$ is abeliangrup.

Proof Truly from the definition 3.2, of additions the 3-D matrices, we see that addition is the sustainable in $\mathcal{M}_{m \times n \times p}(F)$, because

$$
a_{i j k} \in F, b_{i j k} \in F \Rightarrow c_{i j k}=a_{i j k}+b_{i j k} \in F, \forall i=\overline{1, m} ; j=\overline{1, n} ; k=\overline{1, p}
$$

1) Associative property,

$$
\forall \boldsymbol{A}=\left(a_{i j k}\right), \boldsymbol{B}=\left(b_{i j k}\right), \boldsymbol{C}=\left(c_{i j k}\right) \in \mathcal{M}_{m \times n \times p}(F) \Rightarrow(\boldsymbol{A}+\boldsymbol{B})+\boldsymbol{C}=\boldsymbol{A}+(\boldsymbol{B}+\boldsymbol{C})
$$

truly

$$
\begin{aligned}
(\boldsymbol{A}+\boldsymbol{B})+\boldsymbol{C} & =\left[\left(a_{i j k}\right)+\left(b_{i j k}\right)\right]+\left(c_{i j k}\right)=\left(a_{i j k}+b_{i j k}\right)+\left(c_{i j k}\right)=\left(\left(a_{i j k}+b_{i j k}\right)+c_{i j k}\right) \\
& =\left(a_{i j k}+b_{i j k}+c_{i j k}\right)=\left(a_{i j k}+\left(b_{i j k}+c_{i j k}\right)\right)=\left(a_{i j k}\right)+\left(b_{i j k}+c_{i j k}\right)=\boldsymbol{A}+(\boldsymbol{B}+\boldsymbol{C}) \\
\text { 2) } \forall \boldsymbol{A} & =\left(a_{i j k}\right) \in \boldsymbol{M}_{m \times n \times p}(F), \exists \boldsymbol{O}=\left(0_{i j k}\right) \in \mathcal{M}_{m \times n \times p}(F) / \boldsymbol{A}+\boldsymbol{O}=\boldsymbol{O}+\boldsymbol{A}=\boldsymbol{A} .
\end{aligned}
$$
truly,

$$
\begin{aligned}
& \forall \boldsymbol{A}=\left(a_{i j k}\right) \in \mathcal{M}_{m \times n \times p}(F), \exists \boldsymbol{O}=\left(0_{i j k}\right) \in \mathcal{M}_{m \times n \times p}(F) / \boldsymbol{A}+\boldsymbol{O}=\boldsymbol{O}+\boldsymbol{A}=\boldsymbol{A} . \\
& \boldsymbol{A}+\boldsymbol{O}=\left\{\left(a_{i j k}\right)+(0)_{i j k} \mid i=\overline{1, m} ; j=\overline{1, n} ; k=\overline{1, p}\right\} \\
&=\left\{\left(a_{i j k}+0_{F}\right)_{i j k} \mid i=\overline{1, m} ; j=\overline{1, n} ; k=\overline{1, p}\right\} \\
&=\left\{\left(a_{i j k}\right) \mid i=\overline{1, m} ; j=\overline{1, n} ; k=\overline{1, p}\right\}=\boldsymbol{A}
\end{aligned}
$$


3) $\forall \boldsymbol{A}=\left(a_{i j k}\right) \in \mathcal{M}_{m \times n \times p}(F), \exists-\boldsymbol{A}=\left(-a_{i j k}\right) \in \mathcal{M}_{m \times n \times p}(F) / \boldsymbol{A}+(-\boldsymbol{A})=\boldsymbol{O}$.

truly, from Definition 2.4, we have

$$
\begin{aligned}
\boldsymbol{A}+(-\boldsymbol{A}) & =\left\{\left(a_{i j k}+\left(-a_{i j k}\right)\right) \mid i=\overline{1, m} ; j=\overline{1, n} ; k=\overline{1, p}\right\} \\
& =\left\{\left(0_{F}\right)_{i j k} \mid i=\overline{1, m} ; j=\overline{1, n} ; k=\overline{1, p}\right\}=\boldsymbol{O}
\end{aligned}
$$

4) Addition is commutative.

$$
\forall \boldsymbol{A}=\left(a_{i j k}\right), \boldsymbol{B}=\left(b_{i j k}\right) \in \mathcal{M}_{m \times n \times p}(F) / \boldsymbol{A}+\boldsymbol{B}=\boldsymbol{B}+\boldsymbol{A} .
$$

truly

$$
\boldsymbol{A}+\boldsymbol{B}=\left(a_{i j k}\right)+\left(b_{i j k}\right)=\left(a_{i j k}+b_{i j k}\right) \stackrel{(F,+) \text { is abelian }}{=}\left(b_{i j k}+a_{i j k}\right)=\left(b_{i j k}\right)+\left(a_{i j k}\right)=\boldsymbol{B}+\boldsymbol{A}
$$

\section{The "Common" Multiplication of $3 \times 3 \times 3,3$-D Matrices with Elements Froman Field $F$}

Definition 4.1: The multiplication of two matrices $A, B \in \mathcal{M}_{3 \times 3 \times 3}(F)$ we will call the matrix $\boldsymbol{C}=\boldsymbol{A} \otimes \boldsymbol{B} \in \mathcal{M}_{3 \times 3 \times 3}(F)$ calculated as follows.

$\forall\left(\begin{array}{lll}a_{113} & a_{123} & a_{133} \\ a_{213} & a_{223} & a_{233} \\ a_{313} & a_{323} & a_{333} \\ & & \\ a_{112} & a_{122} & a_{132} \\ a_{212} & a_{222} & a_{232} \\ a_{312} & a_{322} & a_{332} \\ & & \\ a_{111} & a_{121} & a_{131} \\ a_{211} & a_{221} & a_{231} \\ a_{311} & a_{321} & a_{331}\end{array}\right)$ 1-vertical la-vertical layer $\left(\begin{array}{ccc}b_{113} & b_{123} & b_{133} \\ b_{213} & b_{223} & b_{233} \\ b_{313} & b_{323} & b_{333} \\ & & \\ b_{112} & b_{122} & b_{132} \\ b_{212} & b_{222} & b_{232} \\ b_{312} & b_{322} & b_{332} \\ & & \\ b_{111} & b_{121} & b_{131} \\ b_{211} & b_{221} & b_{231} \\ b_{311} & b_{321} & b_{331}\end{array}\right) \in \mathcal{M}_{3 \times 3 \times 3}(F)$

The appearance of the multiplication of $3 \times 3 \times 3$, 3D matrices will be as in Figure 4.

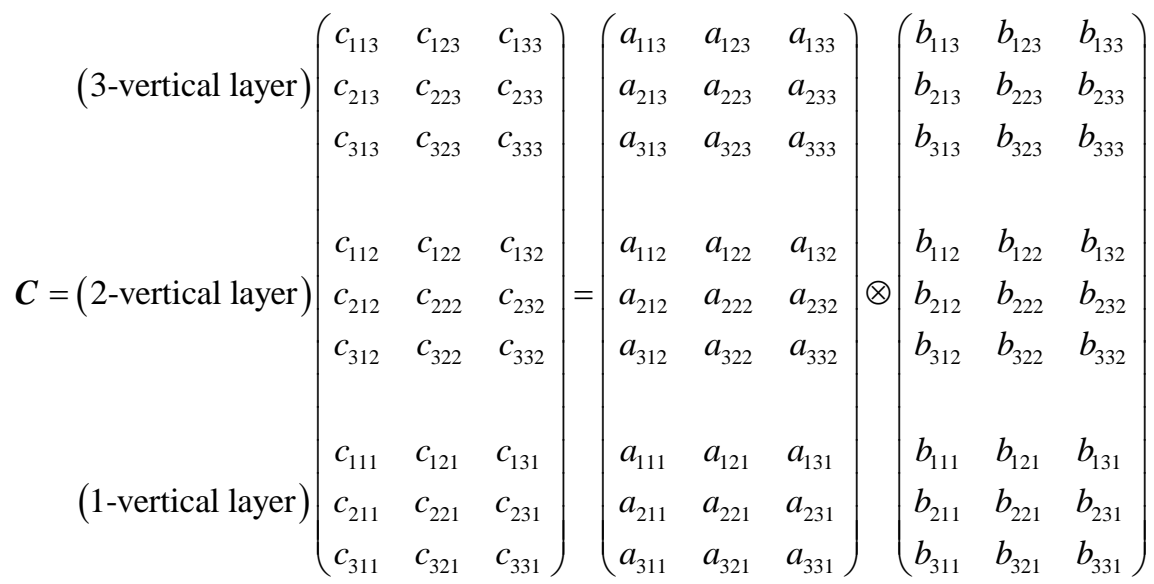

where, the first vertical page is: 


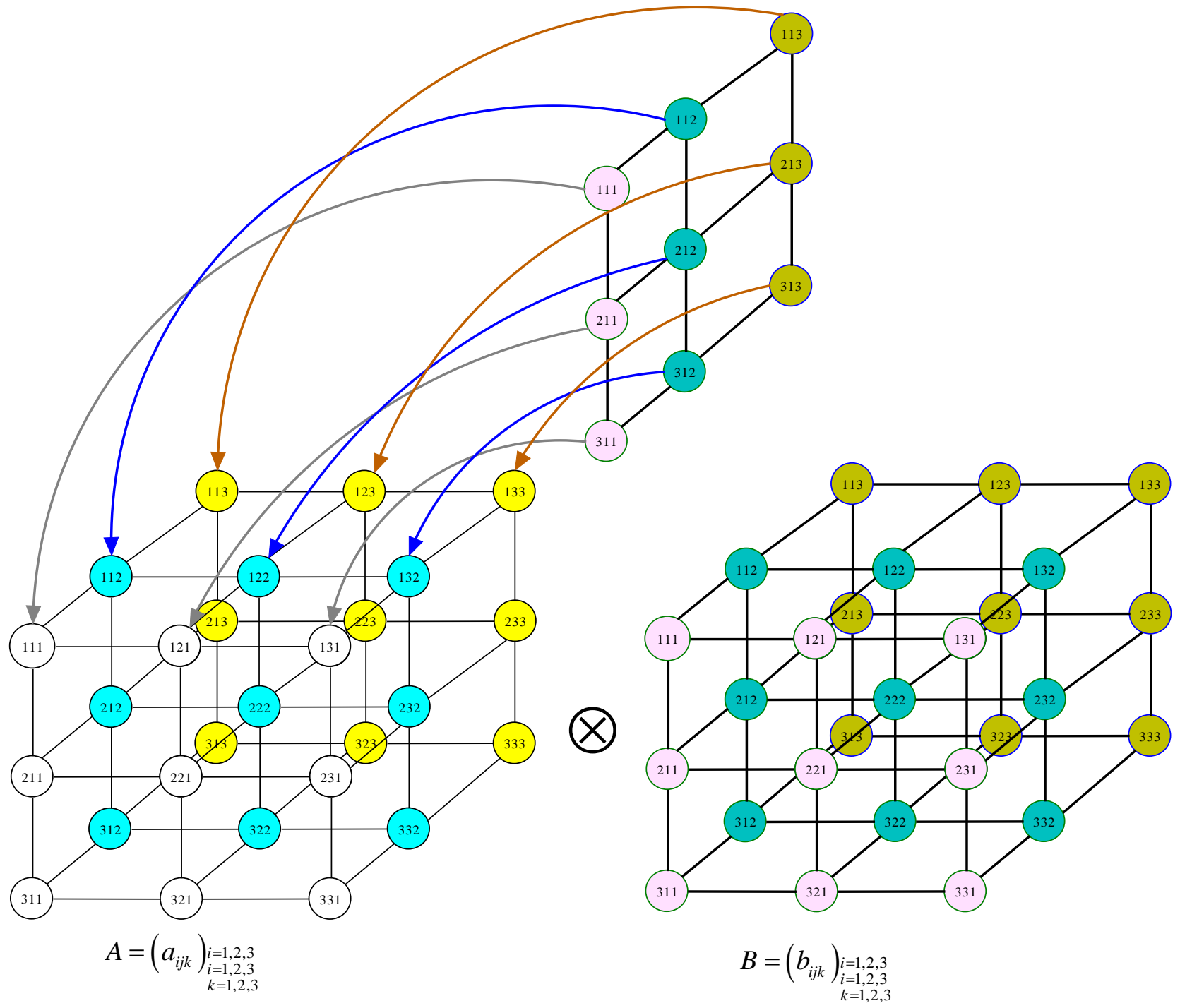

Figure 4. The multiplication of $3 \times 3 \times 3$, 3D matrices.

$$
\begin{aligned}
& c_{111}=a_{111} \cdot b_{111}+a_{121} \cdot b_{211}+a_{131} \cdot b_{311} ; \\
& c_{112}=a_{112} \cdot b_{112}+a_{122} \cdot b_{211}+a_{132} \cdot b_{312} ; \\
& c_{113}=a_{113} \cdot b_{113}+a_{123} \cdot b_{213}+a_{133} \cdot b_{313} ; \\
& c_{211}=a_{211} \cdot b_{111}+a_{221} \cdot b_{211}+a_{231} \cdot b_{311} ; \\
& c_{212}=a_{212} \cdot b_{112}+a_{222} \cdot b_{211}+a_{232} \cdot b_{312} ; \\
& c_{213}=a_{213} \cdot b_{113}+a_{223} \cdot b_{213}+a_{233} \cdot b_{313} ; \\
& c_{311}=a_{311} \cdot b_{111}+a_{321} \cdot b_{211}+a_{331} \cdot b_{311} ; \\
& c_{312}=a_{312} \cdot b_{112}+a_{322} \cdot b_{211}+a_{332} \cdot b_{312} ; \\
& c_{313}=a_{313} \cdot b_{113}+a_{323} \cdot b_{213}+a_{333} \cdot b_{313} ;
\end{aligned}
$$

the second vertical page is:

$$
\begin{aligned}
& c_{121}=a_{111} \cdot b_{121}+a_{121} \cdot b_{221}+a_{131} \cdot b_{321} ; \\
& c_{122}=a_{112} \cdot b_{122}+a_{122} \cdot b_{222}+a_{132} \cdot b_{322} \\
& c_{123}=a_{113} \cdot b_{123}+a_{123} \cdot b_{223}+a_{133} \cdot b_{323} ;
\end{aligned}
$$




$$
\begin{aligned}
& c_{221}=a_{211} \cdot b_{121}+a_{221} \cdot b_{221}+a_{231} \cdot b_{321} ; \\
& c_{222}=a_{212} \cdot b_{122}+a_{222} \cdot b_{222}+a_{232} \cdot b_{322} ; \\
& c_{223}=a_{213} \cdot b_{123}+a_{223} \cdot b_{223}+a_{233} \cdot b_{323} ; \\
& c_{321}=a_{311} \cdot b_{121}+a_{321} \cdot b_{221}+a_{331} \cdot b_{321} ; \\
& c_{322}=a_{312} \cdot b_{122}+a_{322} \cdot b_{222}+a_{332} \cdot b_{322} ; \\
& c_{323}=a_{313} \cdot b_{123}+a_{323} \cdot b_{223}+a_{333} \cdot b_{323} ;
\end{aligned}
$$

and third vertical page is:

$$
\begin{aligned}
& c_{131}=a_{111} \cdot b_{131}+a_{121} \cdot b_{231}+a_{131} \cdot b_{331} ; \\
& c_{132}=a_{112} \cdot b_{132}+a_{122} \cdot b_{232}+a_{132} \cdot b_{332} ; \\
& c_{133}=a_{113} \cdot b_{133}+a_{123} \cdot b_{233}+a_{133} \cdot b_{333} ; \\
& c_{231}=a_{211} \cdot b_{121}+a_{221} \cdot b_{221}+a_{231} \cdot b_{321} ; \\
& c_{232}=a_{212} \cdot b_{122}+a_{222} \cdot b_{222}+a_{232} \cdot b_{322} ; \\
& c_{233}=a_{213} \cdot b_{123}+a_{223} \cdot b_{223}+a_{233} \cdot b_{323} ; \\
& c_{331}=a_{311} \cdot b_{121}+a_{321} \cdot b_{221}+a_{331} \cdot b_{321} ; \\
& c_{332}=a_{312} \cdot b_{122}+a_{322} \cdot b_{222}+a_{332} \cdot b_{322} ; \\
& c_{333}=a_{313} \cdot b_{123}+a_{323} \cdot b_{223}+a_{333} \cdot b_{323} ;
\end{aligned}
$$

It is reduce the above notes through matrix blocks

$$
\left(\begin{array}{l}
\boldsymbol{C}_{3} \\
\boldsymbol{C}_{2} \\
\boldsymbol{C}_{1}
\end{array}\right)=\left(\begin{array}{l}
\boldsymbol{A}_{3} \\
\boldsymbol{A}_{2} \\
\boldsymbol{A}_{1}
\end{array}\right) \otimes\left(\begin{array}{l}
\boldsymbol{B}_{3} \\
\boldsymbol{B}_{2} \\
\boldsymbol{B}_{1}
\end{array}\right)=\left(\begin{array}{l}
\boldsymbol{A}_{3} \times \boldsymbol{B}_{3} \\
\boldsymbol{A}_{2} \times \boldsymbol{B}_{2} \\
\boldsymbol{A}_{1} \times \boldsymbol{B}_{1}
\end{array}\right)
$$

where

$$
\begin{gathered}
\boldsymbol{A}_{1}=\left(\begin{array}{lll}
a_{111} & a_{121} & a_{131} \\
a_{211} & a_{221} & a_{231} \\
a_{311} & a_{321} & a_{331}
\end{array}\right) ; \boldsymbol{A}_{2}=\left(\begin{array}{lll}
a_{112} & a_{122} & a_{132} \\
a_{212} & a_{222} & a_{232} \\
a_{312} & a_{322} & a_{332}
\end{array}\right) ; \boldsymbol{A}_{3}=\left(\begin{array}{lll}
a_{113} & a_{123} & a_{133} \\
a_{213} & a_{223} & a_{233} \\
a_{313} & a_{323} & a_{333}
\end{array}\right) ; \\
\boldsymbol{B}_{1}=\left(\begin{array}{lll}
b_{111} & b_{121} & b_{131} \\
b_{211} & b_{221} & b_{231} \\
b_{311} & b_{321} & b_{331}
\end{array}\right) ; \boldsymbol{B}_{2}=\left(\begin{array}{lll}
b_{112} & b_{122} & b_{132} \\
b_{212} & b_{222} & b_{232} \\
b_{312} & b_{322} & b_{332}
\end{array}\right) ; \boldsymbol{B}_{3}=\left(\begin{array}{lll}
b_{113} & b_{123} & b_{133} \\
b_{213} & b_{223} & b_{233} \\
b_{313} & b_{323} & b_{333}
\end{array}\right) ; \\
\left(\begin{array}{l}
\boldsymbol{C}_{3} \\
\boldsymbol{C}_{2} \\
\boldsymbol{C}_{1}
\end{array}\right)=\left(\begin{array}{l}
\boldsymbol{A}_{3} \\
\boldsymbol{A}_{2} \\
\boldsymbol{A}_{1}
\end{array}\right) \otimes\left(\begin{array}{l}
\boldsymbol{B}_{3} \\
\boldsymbol{B}_{2} \\
\boldsymbol{B}_{1}
\end{array}\right)=\left(\begin{array}{l}
\boldsymbol{A}_{3} \times \boldsymbol{B}_{3} \\
\boldsymbol{A}_{2} \times \boldsymbol{B}_{2} \\
\boldsymbol{A}_{1} \times \boldsymbol{B}_{1}
\end{array}\right)
\end{gathered}
$$

and

$$
\begin{gathered}
\boldsymbol{C}_{1}=\left(\begin{array}{lll}
c_{111} & c_{121} & c_{131} \\
c_{211} & c_{221} & c_{231} \\
c_{311} & c_{321} & c_{331}
\end{array}\right) ; \boldsymbol{C}_{2}=\left(\begin{array}{ccc}
c_{112} & c_{122} & c_{132} \\
c_{212} & c_{222} & c_{232} \\
c_{312} & c_{322} & c_{332}
\end{array}\right) ; \boldsymbol{C}_{3}=\left(\begin{array}{ccc}
c_{113} & c_{123} & c_{133} \\
c_{213} & c_{223} & c_{233} \\
c_{313} & c_{323} & c_{333}
\end{array}\right) \\
\boldsymbol{C}_{1}=\boldsymbol{A}_{1} \times \boldsymbol{B}_{1} ; \boldsymbol{C}_{2}=\boldsymbol{A}_{2} \times \boldsymbol{B}_{2} ; \boldsymbol{C}_{3}=\boldsymbol{A}_{3} \times \boldsymbol{B}_{3}
\end{gathered}
$$

Remark 4.1 Two dimensional matrices can think like matrix with size $m \times n \times 1$

Easy seen from the definition 1, above it that, if $a_{i j 2}=0, a_{i j 3}=0$ and $b_{i j 2}=0, b_{i j 3}=0, \forall i, j \in(1,2,3)$ we get, the usual $3 \times 3$-matrix multiplication, then will take only the first vertical layer is (or, in the language of matrix blocks 
would say that: $\left.\boldsymbol{A}_{2}=0 ; \boldsymbol{A}_{3}=0 ; \boldsymbol{B}_{2}=0 ; \quad \boldsymbol{B}_{3}=0\right)$ :

$$
\left(\begin{array}{llll}
a_{111} \cdot b_{111}+a_{121} \cdot b_{211}+a_{131} \cdot b_{311} & a_{111} \cdot b_{121}+a_{121} \cdot b_{221}+a_{131} \cdot b_{321} & a_{111} \cdot b_{131}+a_{121} \cdot b_{231}+a_{131} \cdot b_{331} \\
a_{211} \cdot b_{111}+a_{221} \cdot b_{211}+a_{231} \cdot b_{311} & a_{211} \cdot b_{121}+a_{221} \cdot b_{221}+a_{231} \cdot b_{321} & a_{211} \cdot b_{121}+a_{221} \cdot b_{221}+a_{231} \cdot b_{321} \\
a_{311} \cdot b_{111}+a_{321} \cdot b_{211}+a_{331} \cdot b_{311} & a_{311} \cdot b_{121}+a_{321} \cdot b_{221}+a_{331} \cdot b_{321} & a_{311} \cdot b_{121}+a_{321} \cdot b_{221}+a_{331} \cdot b_{321}
\end{array}\right)
$$

Definition 4.2. The3-D, unit matrix, associated with the "common" multiplication, must be:

$$
\boldsymbol{I}_{3 \times 3 \times 3}=\left(\begin{array}{ccc}
1 & 0 & 0 \\
0 & 1 & 0 \\
0 & 0 & 1 \\
1 & 0 & 0 \\
0 & 1 & 0 \\
0 & 0 & 1 \\
1 & 0 & 0 \\
0 & 1 & 0 \\
0 & 0 & 1
\end{array}\right) \text { third vertical layer }
$$

or, in the language of matrix blocks:

$$
\boldsymbol{I}_{3 \times 3 \times 3}=\left(\begin{array}{l}
\boldsymbol{I}_{3 \times 3} \\
\boldsymbol{I}_{3 \times 3} \\
\boldsymbol{I}_{3 \times 3}
\end{array}\right)
$$

Easy distinguish that, $\forall \boldsymbol{A} \in \mathcal{M}_{3 \times 3 \times 3}(F) / \boldsymbol{A} \otimes \boldsymbol{I}_{3 \times 3 \times 3}=\boldsymbol{A}$.

Theorem 4.1 $\left(\mathcal{M}_{3 \times 3 \times 3}(F), \otimes\right)$ is a unitary semi-Group with regard to this ordinary multiplication

Proof: 1) associative property. $\forall \boldsymbol{A}, \boldsymbol{B}, \boldsymbol{C} \in \mathcal{M}_{3 \times 3 \times 3}(F)$

$$
\begin{aligned}
& {\left[\left(\begin{array}{l}
\boldsymbol{A}_{3} \\
\boldsymbol{A}_{2} \\
\boldsymbol{A}_{1}
\end{array}\right) \otimes\left(\begin{array}{l}
\boldsymbol{B}_{3} \\
\boldsymbol{B}_{2} \\
\boldsymbol{B}_{1}
\end{array}\right)\right] \otimes\left(\begin{array}{l}
\boldsymbol{C}_{3} \\
\boldsymbol{C}_{2} \\
\boldsymbol{C}_{1}
\end{array}\right)=\left(\begin{array}{l}
\boldsymbol{A}_{3} \times \boldsymbol{B}_{3} \\
\boldsymbol{A}_{2} \times \boldsymbol{B}_{2} \\
\boldsymbol{A}_{1} \times \boldsymbol{B}_{1}
\end{array}\right) \otimes\left(\begin{array}{l}
\boldsymbol{C}_{3} \\
\boldsymbol{C}_{2} \\
\boldsymbol{C}_{1}
\end{array}\right)=\left(\begin{array}{c}
\left(A_{3} \times B_{3}\right) \times C_{3} \\
\left(A_{2} \times B_{2}\right) \times C_{2} \\
\left(A_{1} \times B_{1}\right) \times C_{1}
\end{array}\right)} \\
& \stackrel{\left(\mathcal{M}_{3 \times 3}(F) \times\right) \text { is a semigroup }}{=}\left(\begin{array}{c}
\boldsymbol{A}_{3} \times\left(\boldsymbol{B}_{3} \times \boldsymbol{C}_{3}\right) \\
\boldsymbol{A}_{2} \times\left(\boldsymbol{B}_{2} \times \boldsymbol{C}_{2}\right) \\
\boldsymbol{A}_{1} \times\left(\boldsymbol{B}_{1} \times \boldsymbol{C}_{1}\right)
\end{array}\right)=\left(\begin{array}{c}
\boldsymbol{A}_{3} \\
\boldsymbol{A}_{2} \\
\boldsymbol{A}_{1}
\end{array}\right) \otimes\left[\left(\begin{array}{l}
\boldsymbol{B}_{3} \\
\boldsymbol{B}_{2} \\
\boldsymbol{B}_{1}
\end{array}\right) \otimes\left(\begin{array}{c}
\boldsymbol{C}_{3} \\
\boldsymbol{C}_{2} \\
\boldsymbol{C}_{1}
\end{array}\right)\right] .
\end{aligned}
$$

2) $\exists \boldsymbol{I}_{3 \times 3 \times 3} \in \mathcal{M}_{3 \times 3 \times 3}(F) / \forall A \in \mathcal{M}_{3 \times 3 \times 3}(F) \Rightarrow A \times \boldsymbol{I}_{3 \times 3 \times 3}=A$.

$$
\left(\begin{array}{c}
\boldsymbol{A}_{3} \\
\boldsymbol{A}_{2} \\
\boldsymbol{A}_{1}
\end{array}\right) \otimes\left(\begin{array}{c}
\boldsymbol{I}_{3 \times 3} \\
\boldsymbol{I}_{3 \times 3} \\
\boldsymbol{I}_{3 \times 3}
\end{array}\right)=\left(\begin{array}{c}
\boldsymbol{A}_{3} \times \boldsymbol{I}_{3 \times 3} \\
\boldsymbol{A}_{2} \times \boldsymbol{I}_{3 \times 3} \\
\boldsymbol{A}_{1} \times \boldsymbol{I}_{3 \times 3}
\end{array}\right)\left(\mathcal{M}_{3 \times 3}(F), \times\right) \text { is a unitary semigroup }\left(\begin{array}{c}
\boldsymbol{A}_{3} \\
\boldsymbol{A}_{2} \\
\boldsymbol{A}_{1}
\end{array}\right)
$$

Theorem $4.2\left(\mathcal{M}_{3 \times 3 \times 3}(F),+, \otimes\right)$ is a unitary Ring.

Proof: 1) From Theorem 2.1. $\left(\mathcal{M}_{3 \times 3 \times 3}(F),+\right)$ is abeliangrup.

2) From Theorem 4.1. $\left(\mathcal{M}_{3 \times 3 \times 3}(F), \otimes\right)$ is a unitary semi-Group, and consequently also, $\left(\mathcal{M}_{3 \times 3 \times 3}(F), \otimes\right)$ is a unitary semi-Group

3) $\forall A, B, C \in \mathcal{M}_{3 \times 3 \times 3}(F)$,

a) $\boldsymbol{A} \otimes(B+C)=A \otimes B+A \otimes C . \quad$ b) $(A+B) \otimes C=A \otimes C+B \otimes C$. 
truly

$$
\begin{aligned}
& A \otimes(B+C)=\left(\begin{array}{l}
\boldsymbol{A}_{3} \\
\boldsymbol{A}_{2} \\
\boldsymbol{A}_{1}
\end{array}\right) \otimes\left[\left(\begin{array}{l}
\boldsymbol{B}_{3} \\
\boldsymbol{B}_{2} \\
\boldsymbol{B}_{1}
\end{array}\right)+\left(\begin{array}{l}
\boldsymbol{C}_{3} \\
\boldsymbol{C}_{2} \\
\boldsymbol{C}_{1}
\end{array}\right)\right]=\left(\begin{array}{l}
\boldsymbol{A}_{3} \\
\boldsymbol{A}_{2} \\
\boldsymbol{A}_{1}
\end{array}\right) \otimes\left(\begin{array}{l}
\boldsymbol{B}_{3}+\boldsymbol{C}_{3} \\
\boldsymbol{B}_{2}+\boldsymbol{C}_{2} \\
\boldsymbol{B}_{1}+\boldsymbol{C}_{1}
\end{array}\right)=\left(\begin{array}{c}
\boldsymbol{A}_{3} \times\left(\boldsymbol{B}_{3}+\boldsymbol{C}_{3}\right) \\
\boldsymbol{A}_{2} \times\left(\boldsymbol{B}_{2}+\boldsymbol{C}_{2}\right) \\
\boldsymbol{A}_{1} \times\left(\boldsymbol{B}_{1}+\boldsymbol{C}_{1}\right)
\end{array}\right) \\
& \underset{\left(\mathcal{M}_{3 \times 3}(F),+, \times\right) \text { is a unitary Ring }}{ }\left(\begin{array}{l}
\boldsymbol{A}_{3} \times \boldsymbol{B}_{3}+\boldsymbol{A}_{3} \times \boldsymbol{C}_{3} \\
\boldsymbol{A}_{2} \times \boldsymbol{B}_{2}+\boldsymbol{A}_{2} \times \boldsymbol{C}_{2} \\
\boldsymbol{A}_{1} \times \boldsymbol{B}_{1}+\boldsymbol{A}_{1} \times \boldsymbol{C}_{1}
\end{array}\right)=\left(\begin{array}{l}
\boldsymbol{A}_{3} \times \boldsymbol{B}_{3} \\
\boldsymbol{A}_{2} \times \boldsymbol{B}_{2} \\
\boldsymbol{A}_{1} \times \boldsymbol{B}_{1}
\end{array}\right)+\left(\begin{array}{l}
\boldsymbol{A}_{3} \times \boldsymbol{C}_{3} \\
\boldsymbol{A}_{2} \times \boldsymbol{C}_{2} \\
\boldsymbol{A}_{1} \times \boldsymbol{C}_{1}
\end{array}\right) \\
&=\left(\left(\begin{array}{l}
\boldsymbol{A}_{3} \\
\boldsymbol{A}_{2} \\
\boldsymbol{A}_{1}
\end{array}\right) \otimes\left(\begin{array}{l}
\boldsymbol{B}_{3} \\
\boldsymbol{B}_{2} \\
\boldsymbol{B}_{1}
\end{array}\right)\right)+\left(\left(\begin{array}{l}
\boldsymbol{A}_{3} \\
\boldsymbol{A}_{2} \\
\boldsymbol{A}_{1}
\end{array}\right) \otimes\left(\begin{array}{l}
\boldsymbol{C}_{3} \\
\boldsymbol{C}_{2} \\
\boldsymbol{C}_{1}
\end{array}\right)\right)=\boldsymbol{A} \otimes \boldsymbol{B}+\boldsymbol{A} \otimes \boldsymbol{C} .
\end{aligned}
$$

In a similar manner proved the point (b).

\section{Multiplication of a 3-D, $3 \times 3 \times 3$-Matrix by a Scalar}

Definition 5.1 The multiplication of matrix $A \in \mathcal{M}_{3 \times 3 \times 3}(F)$ with scalar $\lambda \in F$, is matrix $\boldsymbol{C}=\lambda \circ \boldsymbol{A} \in \mathcal{M}_{3 \times 3 \times 3}(F)$ :

$$
\boldsymbol{C}=\lambda \circ\left(\begin{array}{lll}
a_{113} & a_{123} & a_{133} \\
a_{213} & a_{223} & a_{233} \\
a_{313} & a_{323} & a_{333} \\
a_{112} & a_{122} & a_{132} \\
a_{212} & a_{222} & a_{232} \\
a_{312} & a_{322} & a_{332} \\
a_{111} & a_{121} & a_{131} \\
a_{211} & a_{221} & a_{231} \\
a_{311} & a_{321} & a_{331}
\end{array}\right)=\left(\begin{array}{lll}
\lambda \cdot a_{113} & \lambda \cdot a_{123} & \lambda \cdot a_{133} \\
\lambda \cdot a_{213} & \lambda \cdot a_{223} & \lambda \cdot a_{233} \\
\lambda \cdot a_{313} & \lambda \cdot a_{323} & \lambda \cdot a_{333} \\
& & \\
\lambda \cdot a_{112} & \lambda \cdot a_{122} & \lambda \cdot a_{132} \\
\lambda \cdot a_{212} & \lambda \cdot a_{222} & \lambda \cdot a_{232} \\
\lambda \cdot a_{312} & \lambda \cdot a_{322} & \lambda \cdot a_{332} \\
& & \\
\lambda \cdot a_{111} & \lambda \cdot a_{121} & \lambda \cdot a_{131} \\
\lambda \cdot a_{211} & \lambda \cdot a_{221} & \lambda \cdot a_{231} \\
\lambda \cdot a_{311} & \lambda \cdot a_{321} & \lambda \cdot a_{331}
\end{array}\right)
$$

So

$$
\begin{aligned}
\circ: F \times \mathcal{M}_{3 \times 3 \times 3}(F) & \rightarrow \mathcal{M}_{3 \times 3 \times 3}(F) \\
(\lambda, A) & \mapsto \lambda \circ A
\end{aligned}
$$

Theorem $5.1\left(\mathcal{M}_{3 \times 3 \times 3}(F),+,{ }_{F}\right)$ is a vector space

Proof. is evident because, $\left(\mathcal{M}_{3 \times 3}(F),+{ }^{+}{ }_{F}\right)$ it is the vector space, see [6] [8] [9] [10].

Definition 5.2 The multiplication of matrix $\boldsymbol{A} \in \mathcal{M}_{\operatorname{m\times n\times p}}(F)$ with scalar $\lambda \in F$, is matrix $\boldsymbol{C}=\lambda \circ \boldsymbol{A} \in \mathcal{M}_{m \times n \times p}(F)$ :

wherein each element of the matrix is multiplied (by multiplication of the field $F)$ with the element $\lambda \in F$. Well, so we have

$$
\begin{aligned}
\circ: F \times \mathcal{M}_{m \times n \times p}(F) & \rightarrow \mathcal{M}_{m \times n \times p}(F) \\
(\lambda, A) & \mapsto \lambda \circ A
\end{aligned}
$$

Theorem $5.2\left(\mathcal{M}_{m \times n \times p}(F),+, \circ_{F}\right)$ is a vector space

Proof. Is evident because, $\left(\mathcal{M}_{m \times n}(F),+,{ }_{F}\right)$ it is the vector space, see [6] [8] [9] [10] 


\section{Conclusion}

In this article, based on geometric considerations, and mostly considering the cube, we managed to develop the idea of the 3D matrix doing so a generalization of the $2 \mathrm{D}$ matrices, step by step. Furthermore, we gave a unitary ring with the elements of a field F. Initially we gave the ring $3 \times 3 \times 3,3 \mathrm{D}$ matrices and then generalized this concept for $m \times n \times p$, 3D matrices. At the end of this article, we present the scalar multiplication with the $3 \mathrm{D}$ matrices and we show that the set of $3 \times 3 \times 3,3 \mathrm{D}$ matrix, forms a vector space over the field $F$.

\section{References}

[1] Artin, M. (1991) Algebra. Prentice Hall, Upper Saddle River.

[2] Bretscher, O. (2005) Linear Algebra with Applications. 3rd Edition, Prentice Hall, Upper Saddle River.

[3] Connell, E.H. (2004) Elements of Abstract and Linear Algebra.

[4] Schneide, H. and Barker, G.P. (1973) Matrices and Linear Algebra (Dover Books on Mathematics). 2nd Revised Edition.

[5] Lang, S. (1987) Linear Algebra. Springer-Verlag, Berlin, New York.

[6] Nering, E.D. (1970) Linear Algebra and Matrix Theory. 2nd Edition, Wiley, New York.

[7] Zaka, O. and Filipi, K. (2016) The Transform of a Line of Desargues Affine Plane in an Additive Group of Its Points. International Journal of Current Research, 8 34983-34990.

[8] Zaka, O. (2013) Abstract Algebra II. Vllamasi, Tirana.

[9] Zaka, O. (2013) Linear Algebra I. Vllamasi, Tirana.

[10] Zaka, O. (2013) Linear Algebra II. Vllamasi, Tirana.

[11] Zaka, O. and Filipi, K. (2016) One Construction of an Affine Plane over a Corps. Journal of Advances in Mathematics, 12.

\section{Open Access Library}

Submit or recommend next manuscript to OALib Journal and we will provide best service for you:

- Publication frequency: Monthly

- 9 subject areas of science, technology and medicine

- Fair and rigorous peer-review system

- Fast publication process

- Article promotion in various social networking sites (LinkedIn, Facebook, Twitter, etc.)

- Maximum dissemination of your research work

Submit Your Paper Online: Click Here to Submit

Or Contact service@oalib.com 